\title{
1 Eye movements reflect adaptive predictions and predictive precision
}

2 Leah Bakst and Joseph T. McGuire

4 Department of Psychological \& Brain Sciences, Boston University, Boston, MA

5 Center for Systems Neuroscience, Boston University, Boston, MA

6 Mailing address: 677 Beacon Street, Boston, MA 02215

7 Phone number: 617-353-7670

8 Fax number: 617-353-6933

9 Email addresses: lbakst@bu.edu, jtmcg@bu.edu

11 Word count: 7,521

13 Author Note: Some of the data and ideas in this article were presented at the Society for

14 Neuroeconomics annual meeting in 2017, and a preprint was posted on PsyArXiv at

15 https://psyarxiv.com/gh7a5/. The experiment reported in this article was formally preregistered at 16 https://osf.io/sh76b. Raw data (https://osf.io/jdzbq/) and task code (https://osf.io/xezs6/) can be

17 accessed through OSF (Bakst \& McGuire, 2019). Requests for the analysis scripts can be sent

18 via email to the lead author at lbakst@bu.edu.

20 (C) 2020, American Psychological Association. This paper is not the copy of record and may not

21 exactly replicate the final, authoritative version of the article. Please do not copy or cite without

22 authors' permission. The final article will be available, upon publication, via its DOI:

$23 \quad 10.1037 /$ xge0000977 


\section{ABSTRACT}

25 Successful decision making depends on the ability to form predictions about uncertain future

26 events. Existing evidence suggests predictive representations are not limited to point estimates,

27 but also include information about the associated level of predictive uncertainty. Estimates of

28 predictive uncertainty have an important role in governing the rate at which beliefs are updated

29 in response to new observations. It is not yet known, however, whether the same form of

30 uncertainty-modulated learning occurs naturally and spontaneously when there is no task

31 requirement to express predictions explicitly. Here we used a gaze-based predictive inference

32 paradigm to show that (1) predictive inference manifested in spontaneous gaze dynamics; (2)

33 feedback-driven updating of spontaneous gaze-based predictions reflected adaptation to

34 environmental statistics; and (3) anticipatory gaze variability tracked predictive uncertainty in an

35 event-by-event manner. Our results demonstrate that sophisticated predictive inference can occur

36 spontaneously, and oculomotor behavior can provide a multidimensional readout of internal

37 predictive beliefs.

38

39 Keywords: adaptive learning, predictive inference, decision making, eye movements, uncertainty 


\section{INTRODUCTION}

41 Good decision making relies on the ability to make accurate predictions about future events.

42 For instance, your choice of which bus to catch to make an early morning meeting will be guided

43 by a prediction of the time you expect the bus to arrive. Your choice should depend not only on a 44 point prediction (the average or most likely outcome) but also on the associated precision. If the

45 bus's timing is highly variable, you might want to take an earlier bus.

46 Existing evidence supports the idea that decision makers cognitively represent information

47 about the width, not merely the central tendency, of the probability distribution that describes an

48 uncertain future event. Bayesian theories of perception, cognition, and action are premised on the

49 idea that the brain encodes probability distributions to quantify the uncertainty in sensory,

50 cognitive, and motor parameters (Griffiths \& Tenenbaum, 2006; Knill \& Pouget, 2004; Kording

51 \& Wolpert, 2004; Stocker \& Simoncelli, 2006). Maintaining internal probability distributions at

52 intermediate stages of processing can support the adaptive integration of multiple sources of

53 information.

54 Signatures of distributional knowledge are apparent in behavior even when internal beliefs

55 must ultimately be collapsed to a point estimate to select an action or express an overt prediction.

56 In tasks that require explicit predictive estimates, variability across repeated responses is

57 suggestive of a process of sampling from an internal distribution (Bonawitz, Denison, Griffiths,

58 \& Gopnik, 2014; Vul, Goodman, Griffiths, \& Tenenbaum, 2014; Vul \& Pashler, 2008). In tasks

59 that involve sequences of predictions or decisions interleaved with feedback, it has been shown

60 that predictions are adaptively modulated by subjective uncertainty, environmental volatility, and

61 the unexpectedness and predictive validity of new observations (Behrens, Woolrich, Walton, \&

62 Rushworth, 2007; McGuire, Nassar, Gold, \& Kable, 2014; Nassar, Wilson, Heasly, \& Gold, 
63 2010; Nassar, Rumsey, Wilson, Parikh Heasly, \& Gold, 2012; O’Reilly, Schuffelgen, Cuell,

64 Behrens, Mars, \& Rushworth, 2013; Ossmy, Moran, Pfeffer, Tsetsos, Usher, \& Donner, 2013;

65 Payzan-LeNestour \& Bossaerts, 2011; Payzan-LeNestour, Dunne, Bossaerts, \& O’Doherty,

66 2013; Yu \& Dayan, 2005). Internal representations of uncertainty have an important theoretical

67 role in governing belief updating in environments in which observations are noisy and the

68 ground truth is volatile.

69 For instance, a theoretical delta-rule model developed by Nassar and colleagues $(2010 ; 2012$;

70 2016; 2019; McGuire et al., 2014) identifies multiple specific factors via which uncertainty can

71 influence belief updating. One factor in the model ("change-point probability") accounts for

72 people's tendency to adopt a higher learning rate if the previous outcome was extreme relative to

73 their predictive uncertainty. Outcomes outside the predicted range can signal an elevated

74 probability that a change point has occurred in the outcome-generating process and imply that

75 beliefs should be substantially revised. A second factor in the model ("relative uncertainty")

76 accounts for people's tendency to adopt higher learning rates at times when their internal beliefs

77 were more uncertain (relative to their total predictive uncertainty). Incoming evidence is

78 therefore weighted more heavily to refine imprecise beliefs. The model can be extended to

79 account for additional factors that should not normatively influence adaptive learning. For

80 example, empirical data show that learning rates can be elevated by incidental reward (Lee,

81 Gold, \& Kable, 2020; McGuire et al., 2014) and arousal (Nassar et al., 2012), even in contexts in

82 which reward and arousal carry no predictively relevant information. It is not yet clear whether

83 these incidental effects are mediated by representations of uncertainty or other pathways.

84 A key open question is whether uncertainty-modulated predictive inference occurs

85 spontaneously or only in response to specific task demands. The experiments discussed above 
86 relied on tasks in which participants were instructed to make overt predictions or choices. In a

87 number of the studies (e.g. McGuire et al. 2014) the instructions and training included an explicit

88 description of the structure of the outcome-generating process. It is unknown whether people

89 would spontaneously engage in dynamic tracking of predictive uncertainty on the basis of task

90 structure inferred through direct experience. If so, it would imply predictive inference has a

91 highly general role in cognition, consistent with proposals that the brain naturally functions as a

92 predictive engine (Clark, 2013; Summerfield \& De Lange, 2014) and infers statistical regularities

93 from ongoing experience (Griffiths \& Tenenbaum, 2006; Turk-Browne, Scholl, Johnson, \&

94 Chun, 2010).

95 A related methodological question is whether there exists a directly measurable behavioral

96 manifestation of predictive uncertainty. Existing task paradigms have exposed the dynamics of

97 predictive inference by behaviorally eliciting a scalar prediction on each trial (Nassar et al.,

98 2010). These paradigms furnish empirical, event-by-event point estimates of a participant's

99 prediction, the prediction error associated with the subsequent outcome, and the learning rate that

100 guides the ensuing belief update. However, such tasks still require that the width of participants'

101 internal predictive distribution be computationally inferred on the basis of a theoretical model. It

102 is unknown whether the event-specific degree of predictive uncertainty can be measured directly

103 and unobtrusively from behavior.

104 Eye tracking potentially provides a means to address both of the above questions. It is well

105 established that gaze is predictive (Henderson, 2017). For example, eye movements can

106 anticipate the trajectory of a bouncing ball in a manner that evinces a sophisticated predictive

107 model (Diaz, Cooper, \& Hayhoe, 2013; Hayhoe, Mckinney, Chajka, \& Pelz, 2012; Land \&

108 McLeod, 2000). Even more broadly, oculomotor behavior is associated with many latent 
109 cognitive variables. For instance, microsaccades - saccades with amplitudes less than $0.5-1^{\circ}$

110 (Poletti \& Rucci, 2016) - are biased toward the direction of covert spatial attention (Hafed,

111 Lovejoy, \& Krauzlis, 2011; Yuval-Greenberg, Merriam, \& Heeger, 2014), and tend to predict

112 choice (Yu et al., 2016). Gaze behavior has been used extensively to diagnose internal dynamics

113 of decision processes (Cavanagh, Wiecki, Kochar, \& Frank, 2014; Hayhoe \& Ballard, 2005;

114 Konovalov \& Krajbich, 2016; Krajbich, Armel, \& Rangel, 2010; Manohar, Finzi, Drew, \&

115 Husain, 2017; Shimojo, Simion, Shimojo, \& Scheier, 2003).

116 We created an eye-tracking task that implicitly incentivized participants to anticipate the 117 spatial locations of visual targets. Participants were not instructed as to the sequential statistical

118 structure of the task, the optimal strategy, or the behaviors of interest. We designed the

119 experiment to test whether uninstructed, spontaneous predictions would show the same adaptive 120 dynamics that have previously been observed during instructed, overtly expressed predictions,

121 and whether different aspects of oculomotor behavior would reflect the central tendency versus 122 the width of the internal predictive distribution.

123 We hypothesized that gaze position just prior to stimulus onset would be predictive of the 124 upcoming stimulus position, reflecting the central tendency of an internal predictive distribution.

125 To foreshadow our results, this was indeed the case, and such predictions showed evidence for 126 an adaptive learning rate across environments with different generative parameters. We next 127 focused on gaze variability during a pre-stimulus interval, which we hypothesized would be 128 associated with the precision of the predictive distribution. We found that a per-trial measure of 129 pre-stimulus gaze variability was correlated with theoretical levels of uncertainty, both across 130 trials and across environments. Contrary to our expectations, a manipulation of incidental reward 131 had little effect on learning rate or oculomotor dynamics. 
132 Our results suggest that (1) predictive inference manifested in spontaneous gaze dynamics;

133 (2) the updating of gaze-based predictions reflected adaptation to environmental structure; and

134 (3) anticipatory gaze variability carried information about predictive uncertainty. Oculomotor

135 behavior thus appeared to provide a multidimensional readout of internal beliefs, demonstrating

136 the flexibility and generality with which the brain encodes and uses information about predictive

137 uncertainty.

\section{METHODS}

\section{Participants}

140 The study was preregistered with the Open Science Framework (https://osf.io/sh76b; Bakst \& 141 McGuire, 2019). All procedures were approved by the Boston University Institutional Review

142 Board, and informed consent was obtained for all participants. Participants were recruited from

143 the Boston University community ( $\mathrm{N}=56,42$ female, Age: mean $=20.4, \mathrm{SD}=2.0$, range $18-27)$.

144 To determine the appropriate sample size, we performed a power analysis with a significance

145 level of 0.05 , power of 0.8 , and an effect size of $d=0.4$. The sample size required was 51 , which

146 we rounded to the next-higher multiple of 8 based on our counterbalancing scheme (see below).

147 Predicted effects and effect sizes were informed by independent pilot data. All participants had

148 normal or corrected-to-normal vision. One additional participant was excluded based on the

149 preregistered criterion of missing eye-tracking data for more than 100 trials in a given condition

150 (see below). No participants were excluded based on the other preregistered criteria (all

151 completed the full session and had $>60 \%$ task accuracy). A second additional participant was

152 excluded for a reason not anticipated in the preregistration: highly variable eye position in the

153 vertical dimension (standard deviation $>3.5^{\circ}$ ), which was suggestive of poor calibration or other

154 oculomotor issues like nystagmus, given that task stimuli only varied horizontally. 
155 Task

156 Participants performed an implicit spatial prediction task programmed in Python using

157 PsychoPy (v1.85.1, Peirce, 2007). The nominal task was to report whether briefly presented 158 digits were even or odd in order to earn reward. The horizontal position of the digit on the screen 159 varied across trials.

160 The digit on each trial appeared with two flanking Xs for $150 \mathrm{~ms}$ (Figure 1) before being 161 backward-masked by another $\mathrm{X}$, and the participant had unlimited time to respond by pressing 162 "1" with their left hand for "Odd" or "0" with their right hand for "Even." Accuracy feedback (a 163 filled or empty circle) was then displayed for $500 \mathrm{~ms}$ at the same location as the digit, followed 164 by a 750-ms blank inter-trial interval (ITI). The task was designed to create an implicit incentive 165 for participants to anticipate the location of the next digit during the ITI so they could use central 166 vision to make the odd/even judgment.

167 The stimuli on each trial (digit, Xs, and feedback) were presented in either yellow or blue 168 (randomly, with equal probability). One color (counterbalanced across participants) indicated 169 that a correct response would earn one unit of reward (\$0.04), whereas the other color indicated 170 no reward was available. Reward availability was not associated with an outcome's

171 informativeness about future digit locations. Trials were presented in blocks of 40, and a

172 horizontal bar at the bottom of the screen increased in length proportional to the cumulative 173 reward earned in the current block.

174 There were four types of blocks: No, Low, High, and Max Noise. In the Max Noise 175 condition, each digit's horizontal location was drawn independently from a uniform distribution 176 spanning $22.14^{\circ}$ of visual angle ( $42 \%$ of the width of the display). For the No, Low, and High 177 Noise conditions, each digit's horizontal location was drawn from a Gaussian distribution with a 
178 condition-specific standard deviation and a mean that was resampled from the uniform

179 distribution at occasional unsignaled change points (cf. Nassar et al., 2010). The generative mean

180 was not resampled during a two-trial refractory period after each change point, and was

181 resampled with a probability of 0.125 thereafter, leading to an overall average change-point

182 probability of approximately 0.1 . In the No Noise condition, the standard deviation was

183 essentially zero $\left(\sigma<0.01^{\circ}\right)$. In the Low and High Noise conditions, $\sigma=1.67^{\circ}$ and $4.14^{\circ}$,

184 respectively. All stimuli were centered vertically. The condition assigned to the first block of the

185 experiment was systematically counterbalanced across participants; otherwise, the four

186 conditions were sampled in random order without replacement in each set of 4 consecutive

187 blocks.

188 Preliminary training consisted of four 10-trial practice blocks, one of each condition, in order 189 of increasing noise. Participants then completed twenty 40-trial blocks for a total of 800 trials

190 (200 per condition). Participants received no explicit instructions about the different conditions,

191 nor were they told that pre-stimulus gaze position was of interest.

192 Eye tracking acquisition and preprocessing

193 Gaze data were collected monocularly at $1000 \mathrm{~Hz}$ with an EyeLink 1000+ desk-mounted

194 video eye tracker (SR Research Ltd., Osgoode, Canada). Participants used a chin rest positioned $19557 \mathrm{~cm}$ from the display monitor (BENQ XL2430 with a resolution of 1920x1080). Gaze position 196 data were decomposed into saccades, fixations, and blinks using the built-in EyeLink algorithm. 197 The algorithm identified saccades based on the conjunction of three thresholds: position (change $\left.198>0.15^{\circ}\right)$, velocity $\left(>22^{\circ} / \mathrm{s}\right)$, and acceleration $\left(>4000^{\circ} / \mathrm{s}^{2}\right)$. Data from blink periods were excluded 199 from analysis.

200 Spatial prediction from eye position 
Our analyses focused on two summary measures extracted from each trial's gaze time course:

202 (1) predictive gaze position and (2) gaze variability. Predictive gaze position was the horizontal

203 gaze position at the time of digit onset. Because the feedback and ITI had a fixed duration, we

204 hypothesized that gaze position at digit onset would correspond to a prediction of the digit's

205 location. Gaze variability was the amount of eye movement during the 750 ms blank ITI that

206 preceded each trial, which we hypothesized would reflect the degree of uncertainty associated

207 with the upcoming prediction. We originally planned to quantify gaze variability in terms of the

208 per-trial standard deviation of horizontal gaze position across samples. In a deviation from the

209 preregistered plan, we subsequently developed a measure of per-trial gaze variability that

210 factored out the net change in gaze position between the start and end of the ITI in order to avoid

211 a confound between gaze variability and predictive gaze position. The analyses reported below

212 quantify each trial's gaze variability in terms of the total absolute sample-to-sample movement

213 during the ITI minus the absolute value of the net position change (each restricted to the

214 horizontal dimension). Using the originally planned standard deviation metric yielded similar

215 results.

216 Trials with missing data at the time of digit onset were excluded from analyses of predictive

217 gaze position ( $0-68$ trials per participant, mean $=6.3$, median $=0.5)$. Trials with missing data for

218 more than half of the ITI were excluded from gaze variability analyses $(0-83$ trials, mean $=10.9$,

219 median=3.0). The first trial of each block was also excluded, as participants had no basis for a

220 spatial prediction.

221 Comparisons across conditions

222 We used the following general strategy to test for monotonic trends across conditions (in task 223 accuracy, response time, predictive accuracy, gaze variability, saccade and microsaccade 
224 amplitude and frequency). Linear mixed-effect models were fit to the per-subject, per-condition

225 averages. The models included fixed-effect terms for the intercept and slope (as a function of

226 condition, coded 1 through 4 in order of increasing noise), and random-effect terms for per-

227 subject intercepts and slopes. Coefficients and 95\% confidence intervals were estimated using

228 the fitlme function in Matlab. We additionally report Wilcoxon signed-rank tests for each pair of

229 adjacent noise levels, Bonferroni corrected for three comparisons.

230 Theoretical delta-rule model

231 Previous research has shown that a reduced Bayesian model implemented as a delta rule

232 captures key features of dynamic predictive inference (Nassar et al. 2010; 2012; 2019). In brief,

233 the model generates each prediction based on its previous prediction, the prediction error

234 associated with the previous observation, and a dynamically adapted learning rate. The learning

235 rate is itself dependent on estimates of the trial-wise change point probability (CPP) and relative

236 uncertainty (RU). CPP is the probability that the mean of the Gaussian distribution has been

237 resampled, and $\mathrm{RU}$ is the fraction of total predictive uncertainty that is due to imprecise

238 knowledge about the location of the generative mean. Higher values of CPP and RU are

239 associated with higher trial-specific learning rates. (For details of the model see Nassar,

240 McGuire, Ritz, \& Kable, 2019).

241 We estimated per-trial values of CPP and RU from the theoretical model on the basis of the

242 sequence of stimulus locations. We then tested the influence of model-derived factors (per-trial

243 CPP and RU) as well as model-unrelated factors (per-trial reward availability and a condition-

244 specific fixed learning rate) on both belief updating and gaze variability, using a linear regression

245 framework described previously (McGuire et al., 2014). For analyses of belief updating, the

246 dependent variable was the trial-to-trial change in predictive gaze position, and each explanatory 
247 factor was tested in terms of its multiplicative interaction with the previous prediction error. The

248 estimated regression coefficients therefore represented associations between the explanatory

249 factors and learning rate. For analyses of gaze variability, coefficients represented the direct

250 association of each explanatory factor with the empirically observed per-trial gaze variability.

251 Regression models were fit for each participant separately using only data from the Low and

252 High Noise blocks, with coefficients then tested against zero at the group level using Wilcoxon

253 signed rank tests. The belief updating regression model included nuisance terms to account for

254 left-right bias $($ mean $=0.003, p=0.074)$ and edge avoidance $($ mean $=0.011, p<0.001)$.

255 We performed further model robustness checks as exploratory analyses. We first estimated

256 the model-derived CPP and RU values and performed the associated regressions on gaze-based

257 update for each of the four conditions separately (with $\sigma$ set to $0.003^{\circ}, 1.67^{\circ}, 4.14^{\circ}, 6.92^{\circ}$ for No,

258 Low, High, and Max Noise conditions, respectively).

259 We also used a more agnostic approach (Lee et al. 2020) to assess whether larger prediction

260 errors were associated with disproportionately larger (or smaller) updates to gaze-based

261 predictions. We first performed a basic regression for each participant using only an intercept,

262 and a fixed learning rate as regressors for each condition separately. We then combined the

263 residuals across participants and plotted them as a function of prediction error to identify any

264 nonlinear relationships that might be present. We then performed a second regression using an

265 intercept, fixed learning rate, and a nonlinear signed squared prediction error term and overlaid

266 the fit.

267 To simulate the effect of measurement noise on the parameter estimates, we next performed 268 our original regression with the model-derived update (rather than the empirically measured 

specific terms), as well as including a term representing the per-trial total predictive variance (in

273 standard deviation) derived from the model.

A

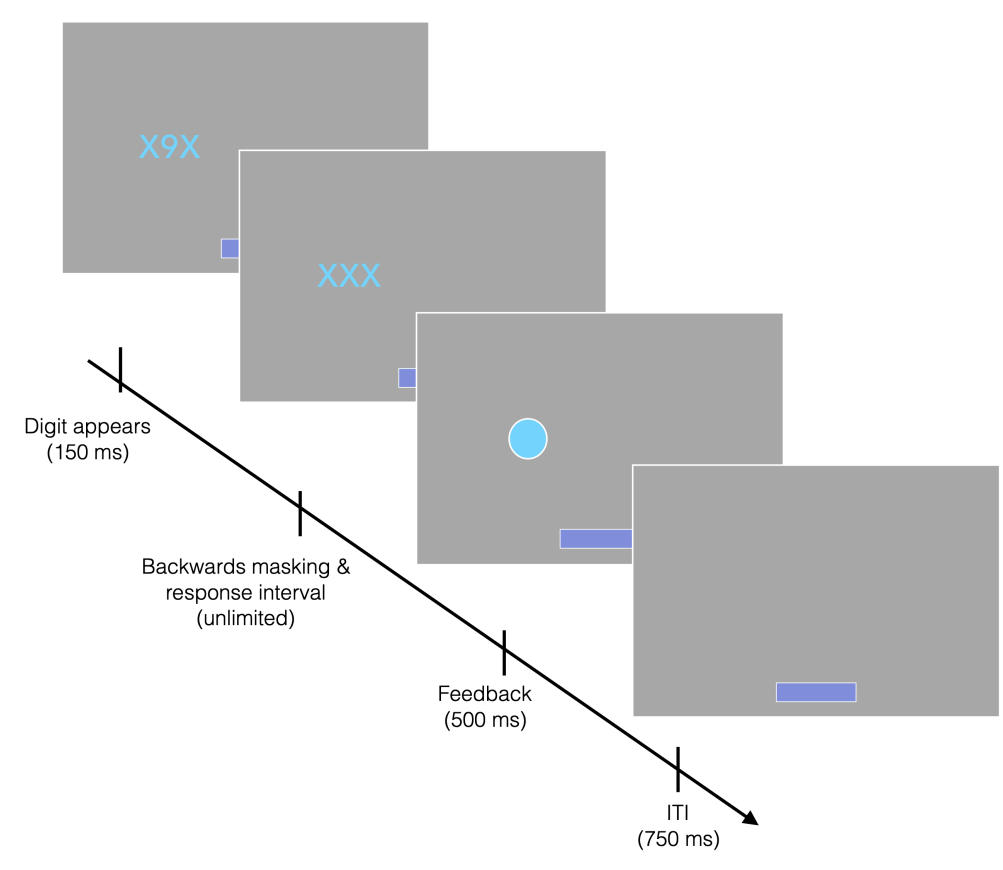

B

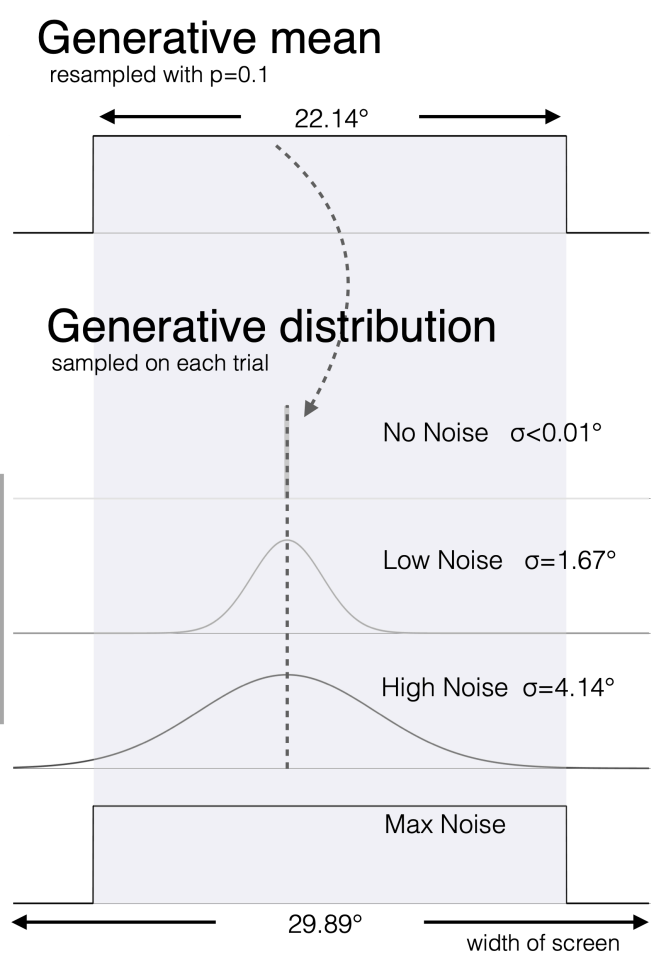

Figure 1. Gaze-based spatial prediction task. (A) A digit was displayed between flanking Xs for $150 \mathrm{~ms}$, before being masked with a third X. The participant had unlimited time to indicate with a key press whether the digit was even or odd. Feedback then appeared for 500 ms: a filled circle indicated a correct response and an unfilled circle indicated incorrect. The ITI was $750 \mathrm{~ms}$. Total reward for each block of 40 trials was represented by the width of the bar at the bottom of the screen. (B) The generative mean was selected from a uniform distribution $22.14^{\circ}$ wide. Gaussian generative distributions in the No, Low, and High Noise conditions had widths of $\sigma<0.01^{\circ}$, $\sigma=1.67^{\circ}$, and $\sigma=4.14^{\circ}$ respectively. Digit locations were selected from these distributions, and the mean was resampled with a probability of 0.1 . In the Max Noise condition, digit locations were selected from the uniform distribution on every trial. 


\section{RESULTS}

285 We used a gaze-based spatial prediction task to test whether spontaneous eye movements 286 carried information about the central tendency and width of internal predictive probability 287 distributions. Participants $(\mathrm{N}=56)$ made even/odd judgments for briefly presented digits (Figure

288 1A-B). The horizontal position of the digit was selected from a Gaussian distribution on each 289 trial. The mean of the Gaussian distribution was resampled at occasional unsignaled change 290 points, whereas the standard deviation (noise) varied across 40-trial blocks. Three levels of noise 291 were used for the Gaussian distribution, resulting in block types that ranged from No Noise (and 292 minimal uncertainty) to High Noise (and high uncertainty). In a fourth condition, Max Noise, 293 digit locations were drawn from a uniform distribution on each trial, to create a maximally 294 unpredictable context. Greater noise theoretically increased the width of the internal predictive 295 distribution by reducing the precision with which the upcoming event could be estimated on the 296 basis of previous observations. 

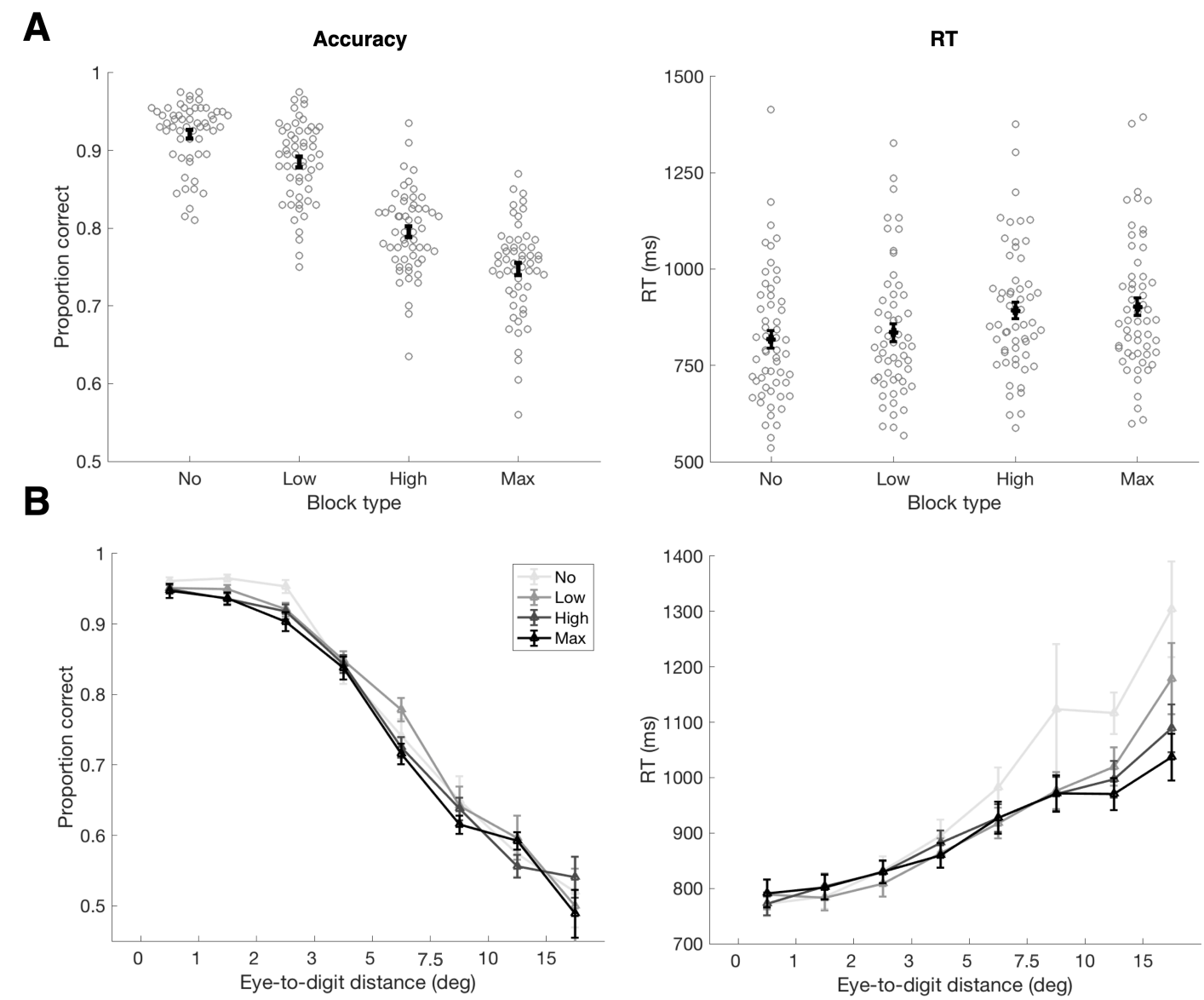

Figure 2. Behavioral performance. (A) Left: Proportion of correct trials for each participant by block type (grey circles, $N=56)$. Group mean $( \pm \mathrm{SEM})$ shown in black. Accuracy decreased with increasing noise level (fixed effect of block type $\beta=-0.06, \mathrm{CI}=-0.066$ to $-0.056, t(55)=-25.39$, $p<0.001$ ); all block types were significantly different in pairwise comparisons (all $p<=0.001$, Wilcoxon signed rank test, Bonferroni corrected for multiple comparisons). Right: Response time (RT) increased with noise level. Within-subject means shown in grey circles, group mean $( \pm$ SEM) shown in black. There was a significant linear trend (fixed effect of block type $\beta=30.92$, $\mathrm{CI}=21.278$ to $40.562, t(55)=6.43, p<0.001)$, though only the Low and High Noise conditions were significantly different using pairwise comparisons with Bonferroni corrections $(p<0.001$; No-versus-Low and High-versus-Max comparisons $p>=0.085)$. (B) Performance as a function of distance between predictive gaze position and the digit. Left shows decreasing accuracy with increasing eye-to-digit distance; right shows increasing RT with increasing eye-to-digit distance. Bins used for eye-to-digit distance were: $0-1^{\circ}, 1-2^{\circ}, 2-3^{\circ}, 3-5^{\circ}, 5-7.5^{\circ}, 7.5-10^{\circ}, 10-15^{\circ}, 15^{\circ}+$. 
310 The overall proportion of correct responses ranged from 0.72 to 0.94 (mean 0.84). Participants

311 tended to respond more accurately and faster when digit locations were highly predictable, and

312 worse, though still above chance, when there was no predictability (Figure 2A). Accuracy

313 depended on the distance between digit and gaze position similarly in all conditions (Figure 2B).

314 Saccade timing suggested participants learned the task's temporal structure (Figure 3). In

315 addition to the saccades evoked by visual stimulus onsets (digit and feedback), saccade

316 frequency increased about 150-200 ms after the beginning of the ITI (highest for Max Noise and

317 lowest for No Noise), and then steadily decreased for the remainder of the ITI until digit onset,

318 consistent with anticipatory stabilization of gaze.

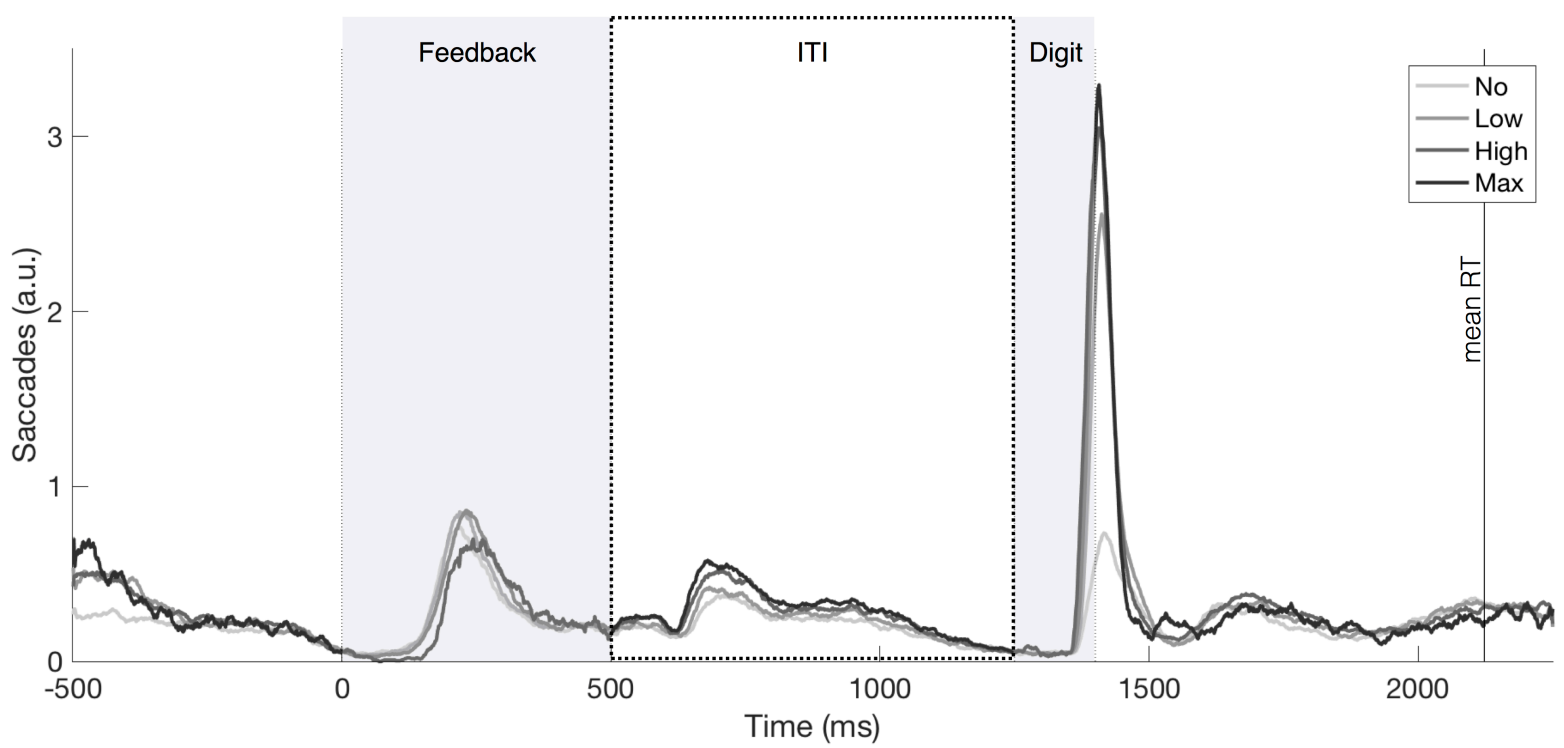

319 Figure 3. Saccades were tallied throughout the trial for each participant, aligned on keypress 320 response time (feedback onset). The saccade histogram was then averaged across the group and 321 smoothed using a 25-ms moving average. The tallies were performed separately for each block 322 type. Analyses of predictive gaze position used gaze at digit onset $(1250 \mathrm{~ms})$. Gaze variability 323 analyses used the ITI (500 to $1250 \mathrm{~ms}$ ). Mean RT ( $873 \mathrm{~ms}$ ) over all participants and block types 324 is shown. 
326 Because digits were presented briefly with crowding and backward masking, the task created

327 an implicit incentive to predict the spatial location of each upcoming digit. Gaze position at the

328 time of digit onset appeared to correspond to a point prediction (Figure 4A). We refer to the

329 horizontal difference between predictive gaze position and the subsequent actual digit location as

330 the "prediction error," and we refer to the difference between predictive gaze position and the

331 true generative mean as "belief error."

332 We evaluated the mean belief error in each condition against a hypothetical experience-

333 independent strategy of always directing gaze to the center of the screen (Figure 4B).

334 Participants consistently outperformed the experience-independent strategy in the No, Low, and 335 High Noise conditions, implying predictive gaze position was dynamically updated on the basis 336 of recent observations. Participants underperformed the experience-independent strategy in Max

337 Noise blocks, consistent with having dynamically updated their predictions even when it was not 338 optimal to do so. 


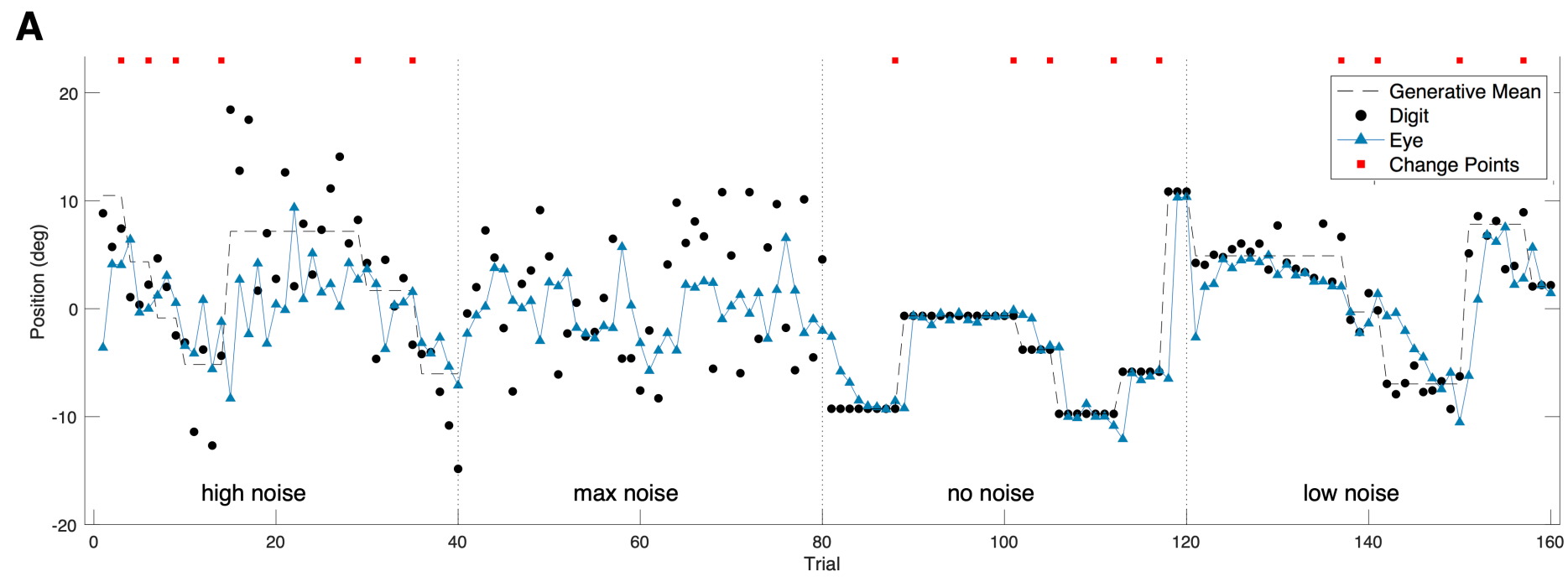

B

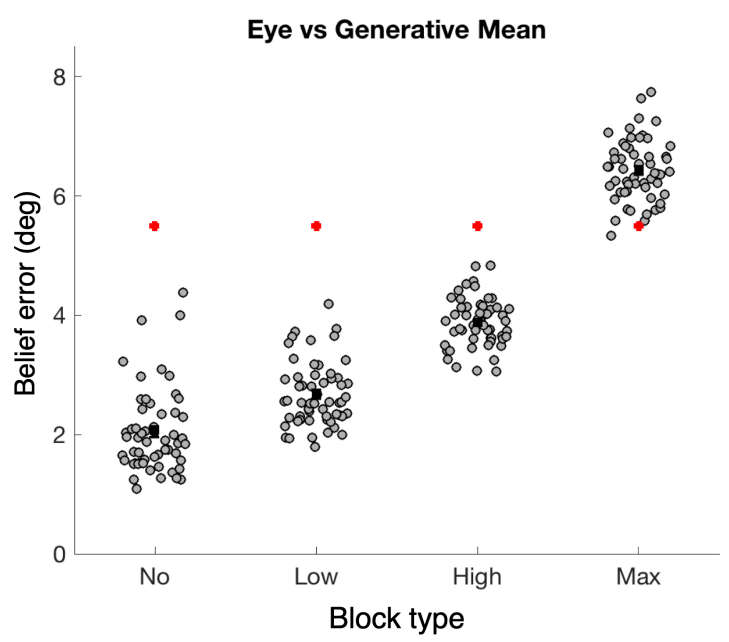

C

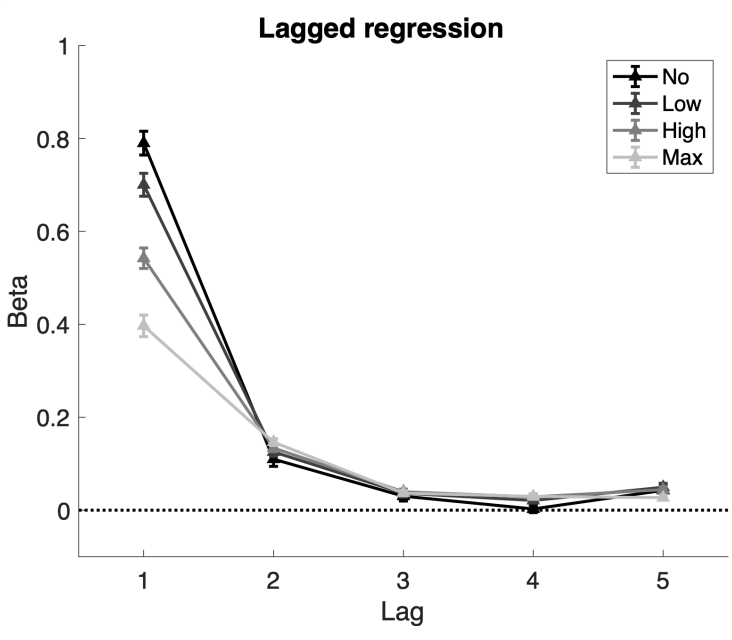

339 Figure 4. Predictive gaze position. (A) Predictive gaze position was defined as the horizontal gaze position at the time of digit appearance. Example data are shown for each block type from a representative participant. Dashed line shows the mean of the generative distribution. Black points show actual digit locations. Blue triangles show predictive gaze position. Red squares indicate change points (changes in the generative mean). (B) Belief error by block type. Grey points indicate the mean difference between the eye and generative mean for each participant. For the Max Noise condition, belief error was equivalent to prediction error (the difference between eye and digit). Group means \pm SEM are shown in black. Error increased with noise level (fixed effect of block type $\beta=1.43, \mathrm{CI}=1.347$ to $1.522, t(55)=32.43, p<0.001$; all pairwise comparisons significantly different $p<0.001$, Wilcoxon signed rank test with Bonferroni correction for multiple comparisons). Red points show the mean expected belief error if the gaze were directed at the screen center. (C) Lagged regression to assess the influence of prior digit locations on the current prediction for each participant. Mean coefficients \pm SEM are shown for five previous trials separated by block type (indicated by hue). All were significantly greater than 354 0.062). 
Predictive gaze position showed evidence of adaptive learning

We next tested whether participants showed evidence of adaptive learning, as distinct from a

357 simple, non-adaptive strategy such as merely leaving their eyes at the location of the previous

358 digit. The time scale of feedback-driven learning can be assessed by testing the relative influence

359 of multiple previous observations on the current prediction (e.g. Corrado, Sugrue, Seung, \&

360 Newsome, 2005). We hypothesized that noisier conditions would favor a prediction that

361 integrated a larger number of observations (Behrens et al., 2007). A lagged regression of

362 predictive gaze position on the five previous observations, pooled across conditions, showed that

363 the greatest weight was given to the most recent digit location but that all five previous trials had

364 decreasing, significantly non-zero weights (all $p<0.001$, Wilcoxon signed rank test).

365 Condition-specific lagged regression analyses showed that participants differentially

366 weighted previous observations depending on the block type, indicative of adaptive learning

367 (Figure 4C). The most recent observation had lower weight in higher-noise conditions than in

368 lower-noise conditions, indicated by significant effects of lag, condition, and their interaction

369 (repeated measures ANOVA, all $\mathrm{F}(1,55)>=179.830$, all $p<0.001$ ). Examining each lag

370 separately, significant effects of condition were seen at lags 1 and 4 (both $F(1,55)>=8.810$, both

$371 p<=0.020$ with Bonferroni correction for multiple comparisons), but not at lags 2, 3 and 5 (all

$372 \mathrm{~F}(1,55)<=5.371$, all $p>=0.120$ with Bonferroni correction). This suggests predictive gaze

373 position reflected an adaptively weighted integration of multiple previous observations.

374 Our next set of analyses examined single-trial learning rates, quantifying the degree to which 375 predictive gaze position was updated relative to the previous prediction error. These analyses

376 focused on the High Noise and Low Noise conditions, which included both random noise and true change-point structure, similar to environments tested in previous studies of overt 
378 predictions (McGuire et al., 2014; Nassar et al., 2010). In an initial exploratory analysis, we 379 quantified the condition-specific average learning rate in terms of the slope of belief update 380 (current minus previous prediction) as a function of prediction error. There were substantial 381 individual differences in learning rate (Figure 5A-B), but learning rates were systematically 382 higher in the Low Noise condition $(p<0.001$, Wilcoxon signed rank test $)$ in addition to being 383 positively correlated between the two conditions across individuals (Spearman $\rho=0.677, p<$

384 0.001). This result was indicative of block-level adaptation of belief updating, consistent with the 385 results of the lagged regression analysis described above. 
A
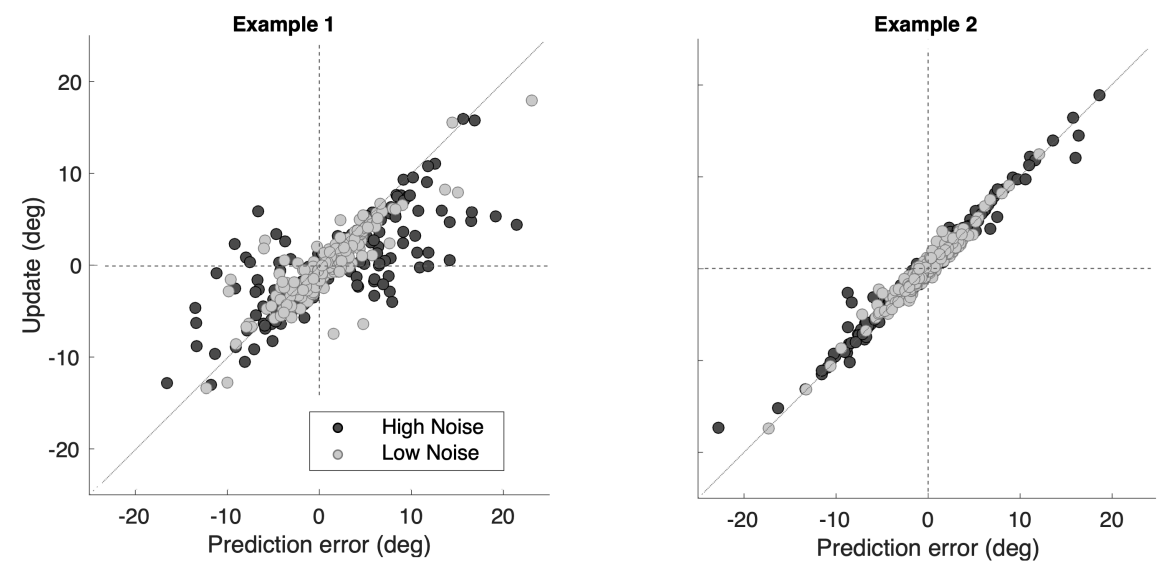

B

C
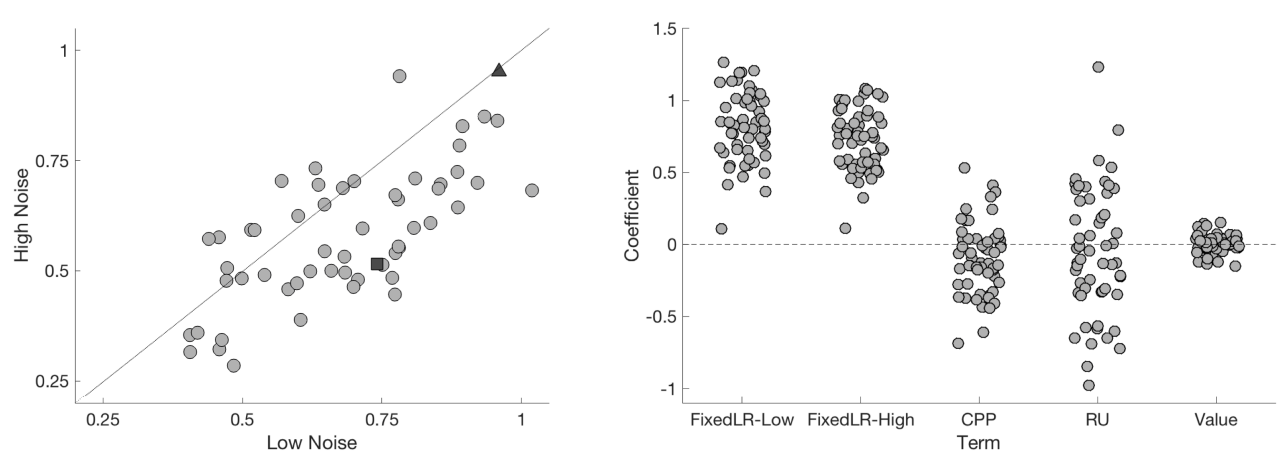

Figure 5. Blockwise and trialwise adaptive learning. (A) Association between prediction error and update (learning rate) in two example participants, for High Noise blocks (dark grey) and Low Noise blocks (light grey). Learning rate could show evidence of adaptation between the High and Low Noise conditions (Example 1) or could be highly linear and non-adaptive (Example 2). Lines were fit separately for the High and Low Noise condition for each participant. Slopes are plotted in (B), with the two examples from Panel A shown in dark grey (Example 1: square, Example 2: triangle). Low Noise slopes were systematically greater than High Noise slopes ( $p<0.001$, Wilcoxon signed rank test), and there was a significant positive correlation between the two conditions (Spearman $\rho=0.677, p<0.001$ ). (C) Coefficients from theoretical model-based regression. Fixed learning rate represents a linear relationship between prediction error and update for Low and High Noise conditions separately. CPP (change point probability) and RU (relative uncertainty) calculated from the delta-rule model (see Methods). Value represents whether or not reward was available on the prior trial. The fixed-learning-rate coefficients differed from zero (both $p<0.001$, Wilcoxon signed rank test), and from each other (Low mean $=0.81$, High mean $=0.72 ; p<0.001$ ). CPP was slightly negative and significantly different from zero $(p=0.004)$, whereas $\mathrm{RU}$ and reward were not (both $p>=0.392$ ). 
To assess trial-by-trial adaptive learning, we applied a theoretical delta-rule model of

403 dynamic predictive inference (Nassar et al. 2010; 2012; 2016; 2019). The model generates a

404 prediction on each trial by integrating the probability that a change point just occurred (change

405 point probability, CPP) with the fraction of total predictive uncertainty due to imprecise

406 knowledge about the location of the generative mean (relative uncertainty, RU) to identify a

407 trial-specific learning rate. The model provides trial-wise normative estimates of CPP and RU on

408 the basis of the sequence of previously observed digit locations, independent of participant

409 behavior. We used a previously developed regression-based approach (McGuire et al., 2014) to

410 estimate the influence of CPP and RU on empirically observed trial-specific belief updating. The

411 regression model also estimated terms for a fixed learning rate for High and Low Noise

412 conditions separately, and the effect of reward availability (Figure 5C).

413 The results yielded evidence for block-level but not trial-level adaptation of learning rates. At

414 the group level, the fixed-learning-rate coefficients for the High Noise and Low Noise conditions

415 significantly differed from zero and from one another (High Noise mean=0.72, Low Noise

416 mean=0.81, $p<0.001$, Wilcoxon signed rank test). Coefficients for CPP were slightly negative

417 on average (and significantly different from zero, $p=0.004$ ), whereas coefficients for RU and

418 reward did not differ from zero (both $p>=0.392$ ).

419 Reward availability has previously been found to influence not only learning rate (Lee et al., 420 2020; McGuire et al., 2014) but also saccade latency and velocity (Manohar et al., 2017). Here, 421 however, a trial's reward availability did not influence the velocity or latency of the first saccade 422 within $300 \mathrm{~ms}$ after digit onset, nor did it influence the duration or dynamics of the subsequent 423 fixation (Supplemental Figure 1). We observed a non-hypothesized effect of block type such that 
424 the post-stimulus saccade tended to be faster (shorter latency and higher velocity) in higher-noise 425 conditions (Supplemental Figure 1).

\section{Gaze variability reflected uncertainty at the block and trial level}

427 We next examined whether gaze variability carried information about the precision of the 428 subjective predictive distribution. We hypothesized that gaze variability during the 750-ms blank 429 ITI prior to each digit's appearance would be elevated (1) in higher-noise blocks relative to 430 lower-noise blocks; and (2) in trials immediately following change points, when participants had 431 the fewest samples of the current generative mean. Gaze variability was quantified in terms of 432 the total horizontal distance traveled from sample to sample, minus the absolute value of the net 433 distance traveled between the start and end of the ITI.

434 Gaze variability was greater during higher-noise blocks (Figure 6A-B). The linear trend and 435 all pairwise differences between conditions were significant at the group level (fixed effect of 436 block type $\beta=0.703, \mathrm{CI}=0.581$ to $0.824, t(55)=11.6, p<0.001$; all pairwise $p<=0.002$,

437 Wilcoxon signed rank test with Bonferroni correction for multiple comparisons). The same 438 finding held if gaze variability was alternatively quantified in terms of the standard deviation of 439 sample-to-sample gaze position within the ITI $(p<0.003$ for all pairwise comparisons, Wilcoxon 440 signed rank test with Bonferroni correction).

441 The effect of condition was largely driven by gaze variability during the first half of the ITI, 442 consistent with the higher frequency of saccades in that period (Figure 3). To assess this, we 443 calculated gaze variability separately for each half of the ITI (1-375 ms and 376-750 ms). In the 444 first half of the ITI, gaze variability increased across conditions (fixed effect of block type $445 \beta=0.494, \mathrm{CI}=0.388$ to $0.600, t(55)=9.36, p<0.001 ; p<=0.002$ for all pairwise comparisons, 446 Wilcoxon signed rank test with Bonferroni correction), whereas the effect was weaker in the 
447 second half of the ITI (fixed effect of block type $\beta=0.139, \mathrm{CI}=0.067$ to $0.211, t(55)=3.87$, $448 p<0.001$; all pairwise comparisons $p>=0.627$, with Bonferroni correction for multiple

449 comparison; Supplemental Figure 2). The linear slope across conditions (e.g. Figure 6A) was 450 significantly greater for the first versus second half of the ITI (Figure 6C; $p<0.001$, Wilcoxon 451 signed rank test).

452 We decomposed gaze variability into four potential contributors — saccade frequency, median 453 saccade amplitude, median microsaccade amplitude, and fixational variability — and found that 454 the first three saccade-related measures increased with noise level across conditions 455 (Supplemental Figure 3), whereas fixational variability did not (Supplemental Figure 4). 456 Gaze variability also transiently increased after change points in the generative mean. Gaze 457 variability increased on the first post-change-point trial in the No, Low, and High Noise 458 conditions, followed by a slow return to baseline over the following trials (Figure 6D). We 459 calculated the Cohen's D effect size for each post-change-point trial for each participant, using 460 the last pre-change-point trial (trial 0) as a baseline (Figure 6E). For the No, Low, and High 461 Noise levels, all mean effect sizes for the first two trials were significantly different from zero 462 (all $p<=0.014$, Wilcoxon signed rank test). Post-change-point increases in gaze variability were 463 more strongly apparent in the first half than the second half of the ITI (Supplemental Figure 2). 464 We conducted an exploratory analysis using the regression framework introduced above to 465 test trial-by-trial associations between gaze variability and theoretical model-derived factors in 466 the High Noise and Low Noise conditions. The analysis showed significant effects of RU and 467 CPP (Figure 6F, both $p<0.001$, Wilcoxon signed rank test), consistent with the post-change468 point increase in gaze variability identified above. We found a marginal effect of the prior trial's 
different intercept coefficients (Low Mean $=8.21$, High Mean $=8.78, p<0.001)$.

A

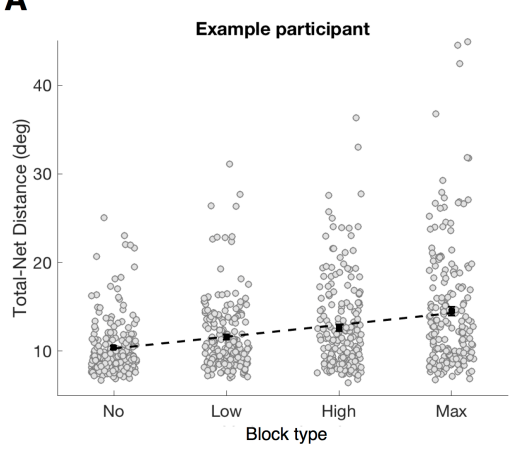

D

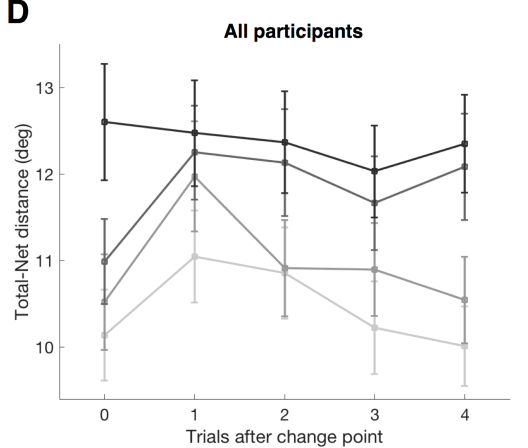

B

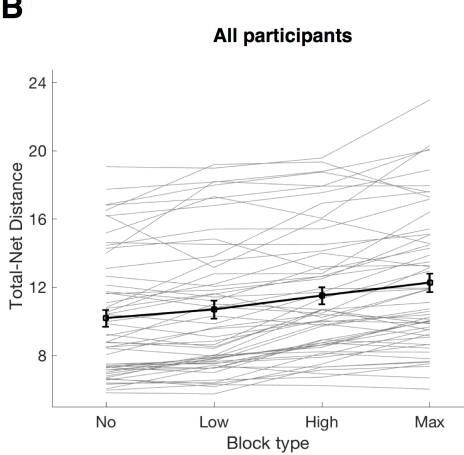

E

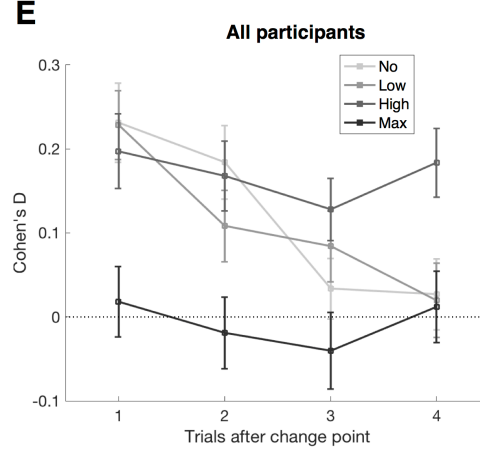

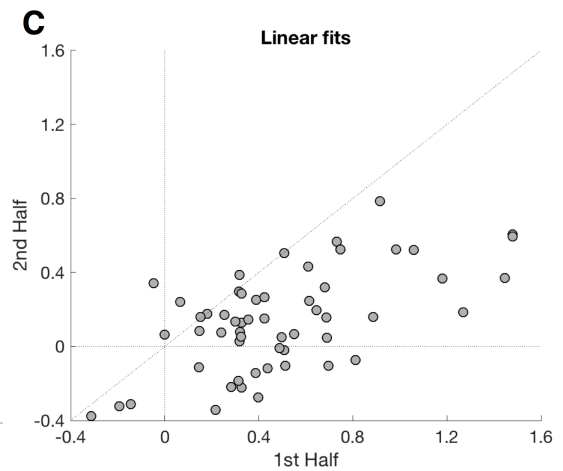

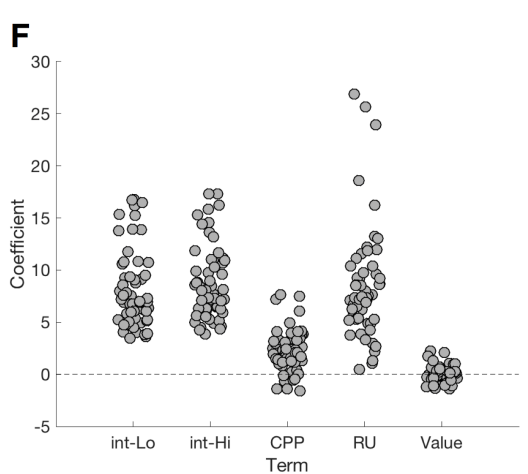

471

472

473

474

475

476

477

478

479

480

481

482

483

484

485

486

487

488

489

490

491

Figure 6. Gaze variability as a readout of predictive precision. (A) Example data from one participant. Grey circles represent trialwise gaze variability during the ITI. Means \pm SEM are in black. Dashed line is linear fit. (B) Gaze variability for all participants. Individual data shown in grey, group mean \pm SEM in black. Gaze variability increased with noise level (fixed effect of block type $\beta=0.703$, CI 0.581 to $0.824, t(55)=11.6, p<0.001$; all pairwise comparisons significantly different $p<=0.002$, Wilcoxon signed rank test with Bonferroni correction). (C) Linear slopes fit to each participant's gaze variability across the four conditions, for each half of the ITI separately. The slope from the first half of the ITI was significantly greater than from the second ( $p<0.001$, Wilcoxon signed rank test). (D-E) Gaze variability aligned on change points. (D) Gaze variability \pm SEM separated by noise level (indicated by the hue), aligned on change points. Gaze variability on trial zero was calculated before the digit appeared in the resampled location. Max Noise blocks lacked true change point structure; data are shown aligned to arbitrarily designated change point trials for comparison. (E) Effect size for each post-changepoint trial relative to trial zero. Cohen's D was calculated for each participant for each noise level, and the mean \pm SEM is shown. For the No, Low, and High Noise levels, all effect sizes were significantly different from zero for the first two trials (all $p<=0.014$, Wilcoxon signed rank test). (F) Coefficients from theoretical model-based regression with gaze variability as dependent variable. The coefficients for both intercepts, RU, and CPP were significantly different from zero (all $p<0.001$, Wilcoxon signed rank test). The intercept terms differed between the Low-Noise and High-Noise conditions (Low mean $=8.21$, High mean $=8.78, p<$ 0.001, Wilcoxon signed rank test). 

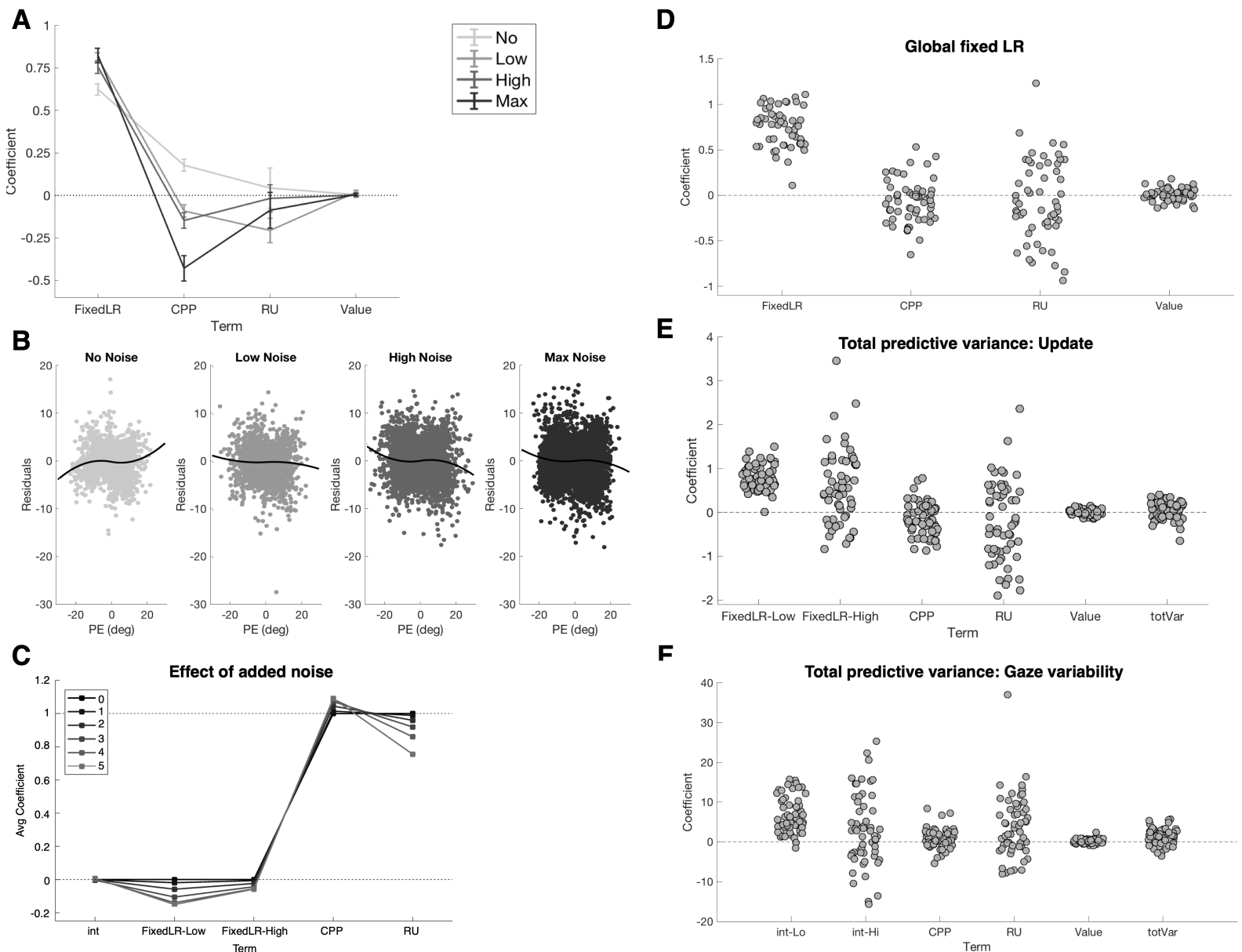

Figure 7. Model robustness checks. (A) The analysis of belief updating was performed for each participant separated by condition. Mean coefficient across subjects and standard error are shown. Fixed-learning-rate coefficients (fixed LR) were significantly different between the No, Low, and High noise conditions ( $p<=0.038$, Wilcoxon signed rank test). CPP was significantly greater than zero only for the No Noise condition $(p<0.001)$, and significantly less than zero in the other conditions ( $p<=0.019$ ). Only the Low Noise condition showed a significant (albeit negative) effect of RU ( $p=0.004$; all other $p>=0.231)$. No condition showed any significant load on reward value ( $p>=0.105)$. (B) To visualize a possible nonlinear relationship between prediction error and update, an initial regression was performed with an intercept and fixed LR as regressors for each subject, separated by condition. The residuals from that regression were combined across subjects and plotted against their associated prediction errors. A second regression was then performed with a fixed LR term and a nonlinear term (the signed squared prediction error), and its fit was overlaid (thick black line). (C) A regression was performed on the theoretical model-generated update combined with various levels of Gaussian noise ( $\sigma=0$ to $5^{\circ}$ ), using the same explanatory variables as the original regression. (D) Regression using one global fixed LR regressor instead of condition-specific regressors. Fixed LR was significantly greater than zero (mean $=0.74, p<0.001$, Wilcoxon signed rank test). CPP was slightly negative on average $(-0.07, p=0.022)$. Both RU and reward were not significantly different from zero $(p$ $>=0.344)$. (E) Total predictive variance was added as a regressor with update as the outcome variable. Both condition-specific fixed LR terms were significantly greater than zero $(p<0.001)$. CPP was slightly less than zero $(p=0.006)$, while RU was negative on average but statistically 
513 indistinguishable from zero $(p=0.081)$. Neither reward nor total predictive variance ("totVar") 514 was significantly different from zero $(p>=0.091)$. (F) Total predictive variance was also added 515 as a regressor with gaze variability as the outcome variable. Coefficients for CPP, RU and total

516 variance were significantly greater than zero (all $p<=0.011$ ). Reward had a very small positive

$517 \operatorname{effect}(p=0.031)$.

518 Model robustness checks

519 The observed trial-level modulation of gaze variability is seemingly at odds with the lack of

520 evidence for trial-level adaptation in the rate of belief updating. We conducted a series of

521 exploratory analyses and robustness checks to probe the pattern of results further. First, we

522 performed a regression analysis of predictive gaze for each of the four conditions individually

523 (Figure 7A). Results differed systematically across conditions. The No Noise condition showed

524 significant positive load on CPP as well as on the fixed learning rate component $(p<0.001$,

525 Wilcoxon signed rank test), implying participants adaptively modulated their learning rate at the

526 trial level in that condition. The Low and High Noise conditions both showed stronger weight on

527 the fixed LR component compared to No Noise $(p<=0.005)$, and a slightly negative weight on

528 CPP $(p<=0.019)$. In contrast, the Max Noise condition had a larger negative weight on CPP $(p<$

529 0.001). This suggests that when participants observed particularly large prediction errors in

530 higher-noise conditions, they updated their prediction using a lower learning rate, treating

531 extreme events as if they represented noise instead of meaningful change.

532 To probe the relationship between prediction error and update in a model-agnostic manner,

533 we used an approach from Lee, Gold \& Kable (2020). We first performed a basic regression for

534 each participant using only an intercept and a fixed learning rate as regressors for each condition

535 separately. We then combined the residuals across participants and plotted them against the prior

536 prediction error to highlight any nonlinear effects that had not been regressed out (Figure 7B).

537 We then performed a second regression using an intercept, prediction error, and a nonlinear

538 signed squared prediction error term, and overlaid the fit. The more agnostic approach confirmed 
that the No Noise condition showed nonlinear effects consistent with adopting a higher learning rate for extreme events, whereas the other three conditions show varying levels of nonlinear

541 effects in the opposite direction, consistent with adopting a lower learning rate for extreme 542 events.

543 We next sought to verify that the lack of expected trial-level effects in learning rate 544 adaptation in the Low and High Noise conditions was not due to the particularities of our model. 545 We implemented an additional three versions of the model (Figure 7C-F): (1) a regression using 546 the normative model output; (2) global fixed learning rate; (3) total predictive variance.

547 To evaluate the potential effects of measurement noise on the estimated coefficients, we 548 performed the same regression using the theoretical model-derived update as the dependent 549 variable (Figure 7C). Even with Gaussian noise ( $\sigma$ ranged from $0^{\circ}$ to $5^{\circ}$ ) added to the update, the 550 load on the fixed-learning-rate terms for both Low and High noise did not approach levels 551 observed in the empirical data, and the analysis recovered strongly positive coefficients for CPP 552 and RU. This result demonstrates that effects of CPP and RU could theoretically have been 553 detected using our analyses methods.

554 Next we examined whether condition-specific learning rate terms in the original analysis 555 might have captured variance attributable to CPP or RU (Figure 7D). In a variant of the 556 regression analysis that included only one fixed learning rate parameter, there remained a 557 significant load on the fixed learning rate $(p<0.001$, Wilcoxon signed rank test), no significant 558 effect of RU ( $p=0.344)$, and a slightly negative effect of CPP ( $p=0.022)$, suggesting the 559 condition-specific terms had not obscured trial-level effects.

560 Finally, we implemented a model that included a term for per-trial estimates of total 561 predictive variance derived from the theoretical delta-rule model (Figure 7E). Though total 
562 predictive variance is related to RU, the predictor matrix was full-rank indicating that the two

563 were not linearly dependent. The coefficients associated with total predictive variance were not

564 significantly different from zero $(p>=0.071)$, although the term did tend to improve the fit of the

565 model as a whole: $Z$-transformed $F$ statistics were computed for the nested model comparison

566 within each individual, and were significantly greater than zero at the group level $(p<0.001$,

567 Wilcoxon signed rank test).

568 In a similar way, we tested the effect of model-derived total predictive variance on gaze

569 variability (Figure 7F). Given that we had already observed a relationship between gaze

570 variability and components of predictive uncertainty (Figure 6), we expected we might identify

571 an effect of total predictive variance as well. The results showed that total predictive variance,

$572 \mathrm{CPP}$, and RU all had significantly positive coefficients (all $p<=0.011$ ), implying all three

573 factors were associated with unique variance in per-trial anticipatory gaze variability. This

574 finding suggests total predictive variance may be a cognitively meaningful factor

575 notwithstanding its lack of any systematic effect on belief updating (Figure 7E).

576 Optimality and strategy differences across individuals

577 Previous studies have observed large individual differences in participants' tendency to adopt

578 successful strategies for adaptive learning (McGuire et al., 2014; Nassar et al., 2010; 2012;

579 2016). We tested whether the optimality of participants' belief-updating strategies was related to

580 three indices of gaze variability: (1) average overall gaze variability; (2) gaze variability slope

581 across block types (e.g. Figure 6A); and (3) Cohen's D effect size for the first post-change-point

582 trial versus baseline in the Low Noise condition (e.g. Figure 6E).

583 We originally hypothesized that the three indices of gaze variability would be associated with

584 two indices of the optimality of gaze-based predictions. The first optimality index was overall 
585 learning rate, calculated as the slope of update as a function of prediction error across all trials

586 (e.g. Figure 5A); based on previous work, we predicted that lower learning rates would track

587 better performance (McGuire et al., 2014; Nassar et al., 2010). In fact, however, the relationship

588 between average learning rate and predictive accuracy in our data set was less straightforward,

589 and accordingly the average learning rate showed no significant associations with gaze

590 variability indices (all $p>=0.151$ ). The second planned optimality index was the sum of the CPP

591 and RU coefficients from the theoretical model-based regression analysis of belief updating

592 (Figure 5C). Consistent with the null effects of trial-level factors on learning rate, that index also

593 showed no associations with gaze variability (all $p>=0.176$ ).
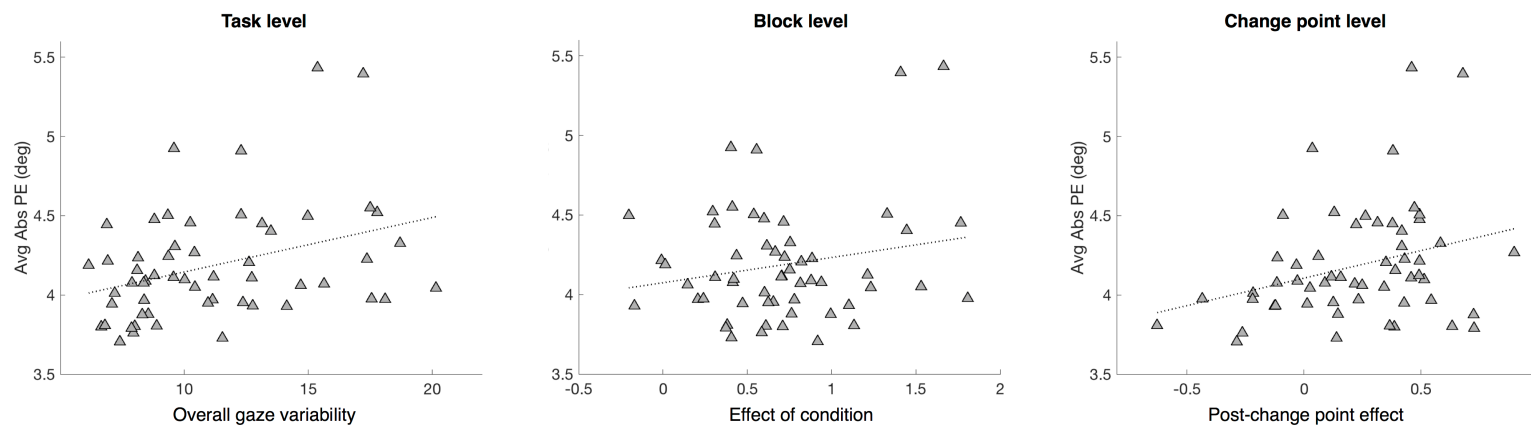

594

595

596

597

598

599

600

601

602

603

604

605

Figure 8. Individual differences in optimality. The mean absolute prediction error for each participant was compared to three indices of gaze variability: (1) Left: Mean overall gaze variability within the ITI $\left(\mathrm{R}^{2}=0.124, p=0.008\right)$, (2) Center: Slope of a line fit to gaze variability across conditions $\left(\mathrm{R}^{2}=0.039, p=0.143\right),(3)$ Right: Cohen's $\mathrm{D}$ effect size for the first trial after the change point in the Low Noise condition $\left(\mathrm{R}^{2}=0.090, p=0.025\right)$.

We performed an exploratory analysis in which we compared mean absolute prediction error, an inverse measure of the optimality of gaze-based predictions, with the three gaze variability indices (Figure 8). We observed positive correlations with overall variability and post-changepoint effects (all $\mathrm{R}^{2}>=0.090$, all $p<=0.025$ ), whereas the slope of gaze variability across conditions was positive but not significant $\left(\mathrm{R}^{2}=0.039, p=0.143\right)$. This pattern implies, contrary to our original hypothesis, that larger uncertainty-related changes in gaze variability were associated with less successful performance. 


\section{DISCUSSION}

The present study investigated whether spontaneous, gaze-based predictions would show the

608 same adaptive learning dynamics that have previously been observed for explicit predictions

609 (McGuire et al., 2014; Nassar et al., 2010, 2012, 2016, 2019; O’Reilly et al., 2013). We found

610 evidence that anticipatory gaze position corresponded to the central tendency of participants'

611 predictive beliefs, whereas gaze variability during the pre-stimulus interval corresponded to

612 belief precision. We found evidence that participants adapted their learning rate across blocks,

613 but we did not observe the trial-level adaptation seen in previous studies involving explicitly

614 elicited predictions. Gaze variability showed evidence of both block-level and trial-level

615 adaptation.

616 Point predictions

617 Gaze position at the time of digit onset appeared to reflect the central tendency of

618 participants' internal predictive probability distribution. The prediction showed evidence of

619 learning (Figure 4B), and integrated multiple previous observations using an adaptive learning

620 rate across conditions (Figure 4C). Gaze-based predictions did not show evidence of the

621 expected trial-level adaptation of learning rate, which differed from previous results in the

622 context of explicit prediction (McGuire et al., 2014). Overall, this set of findings suggests people

623 spontaneously track predictive uncertainty, and supports a highly general role for predictive

624 inference in cognition.

625 One potential explanation for the absence of trial-level adaptation is that participants may

626 have spontaneously reached a partially inaccurate inference about the task's generative structure.

627 In reality, the sequence of outcomes was governed by a combination of Gaussian observation

628 noise (a source of expected uncertainty) and occasional, uniformly distributed instances of 
629 volatility in the generative mean (a source of unexpected uncertainty). Previous studies that

630 elicited overt predictions in similarly structured environments - and explicitly described the

631 generative structure to participants - have shown evidence that people adaptively used a higher

632 learning rate when outcomes were more surprising or when their beliefs were less precise (e.g.

633 Nassar et al., 2016). However, studies that provided training with other types of generative

634 structure have observed different behavior. Participants correctly adopted a fixed learning rate

635 when volatility in the generative mean was governed by smooth drift (Lee et al., 2020), and

636 downregulated their learning rate for extreme events when such events were uninformative

637 outliers rather than true change points (D'Acremont \& Bossaerts, 2016; Nassar, Bruckner, \&

638 Frank, 2019; O’Reilly et al., 2013).

639 A possibility, therefore, is that participants in the present study did not spontaneously tend to

640 infer change-point-like structure from ambiguous sequences of experiences. Participants did

641 show some evidence of having adapted their learning rate in the expected manner in the No

642 Noise condition, where change points were the most obvious (Figure 7A), but in the other

643 conditions they might instead have inferred a generative mechanism characterized by continuous

644 drift or uninformative outliers. An incorrect structural inference could explain why participants

645 might have registered trial-by-trial variability in predictive uncertainty (as suggested by our gaze

646 variability analyses) without translating it into the expected trial-by-trial adaptive learning rate.

647 Future studies should further examine factors that influence feedback-driven inferences about

648 generative structure in the absence of explicit description. For example, the present task used

649 relatively short 40-trial blocks, and included one condition (Max Noise) that lacked change point

650 structure. It is possible more sustained experience with stable generative statistics would cause

651 different learning rate dynamics to emerge. In addition, future work should investigate dynamic 
652 predictive inference in the context of qualitatively different forms of generative structure. For

653 instance, a direct comparison between change-point-like structure and continuous drift with

654 uninformative outliers would help determine whether participants tend to make inaccurate

655 inferences about particular task structures, or if there is a limit to their learning and accuracy in

656 spontaneous predictive inference more generally. This kind of experiment will be critical for

657 determining what structural models participants adopt spontaneously, and how structural

658 assumptions interact with feedback-driven learning.

659 Gaze variability

660 Gaze variability during the pre-stimulus interval was inversely related to predictability at the

661 scale of both blocks (Figure 6B) and trials (Figure 6D). A potential interpretation is that

662 oculomotor behavior carries information about the width of the underlying internal predictive

663 distribution. The result raises several questions worth investigating in future research.

664 First, what is the mechanistic link between gaze variability and predictive uncertainty?

665 Recent work on the link between attention and the variability of neuronal activity suggests one

666 potential explanation. Attention tends to decrease the variability of activity in single neurons

667 (Luo \& Maunsell, 2018; Nandy, Nassi, \& Reynolds, 2017; von Trapp, Buran, Sen, Semple, \&

668 Sanes, 2016). In our task, participants directed overt attention to the predicted location of the

669 next digit, and may have allocated covert attention to a range of locations proportional to the

670 uncertainty in the prediction. A wider attentional window could be associated with greater

671 variability in neural activity, which could propagate to the generated oculomotor commands and

672 result in a more variable eye position.

673 Increased gaze variability could also be a strategy in its own right. It could represent a

674 process of sampling before converging on a single location to fixate. If the system initially 
675 sampled a region proportional to the amount of uncertainty, gaze variability would be expected 676 to increase with uncertainty, especially at early time points. Accordingly, we found that the

677 correspondence between gaze variability and uncertainty was observed primarily in the first half 678 of the ITI (Figure 6C).

679 A second question has to do with which specific cognitive factor is most strongly reflected in 680 gaze variability. Gaze variability appeared to decouple from learning rate, and tracked trial-by681 trial changes in uncertainty that were not reliably reflected in learning dynamics. High gaze

682 variability in the Max Noise condition suggests gaze variability indexed uncertainty either in the 683 current estimate of the generative mean or in the predicted location of the upcoming target. A 684 potentially incongruous observation is that gaze variability increased after change points in the 685 No Noise condition when beliefs and predictions should have been relatively precise. The fact 686 that the post-change-point increase in gaze variability was smallest in the No Noise condition 687 suggests gaze variability was not merely related to the size of the previous prediction error. An 688 increase in uncertainty on those trials could have come about if participants inferred an imprecise 689 model of the task structure.

690 A third question relates to the extent to which oculomotor behavior in some way represents 691 the full shape of the internal belief distribution. Future work using additional classes of 692 distributions (e.g. skewed, multimodal, etc.) will be essential in answering this question. It 693 remains to be determined whether gaze variability represents a scalar factor like predictive 694 uncertainty or, alternatively, carries additional information about the shape of the subjective 695 probability distribution akin to a sampling process.

696 Individual differences 
Previous work has shown that individuals vary in the extent to which they exhibit optimally 698 adaptive learning (McGuire et al., 2014; Nassar et al., 2010, 2012). Contrary to our expectations,

699 more optimal behavior in our task was generally associated with weaker oculomotor effects

700 (Figure 7). Because the four conditions differed considerably in their governing statistics,

701 participants might have optimized their behavior for only a subset of the conditions they

702 experienced, making it challenging to identify and detect overall signatures of optimal behavior.

703 For example, although participants adapted their learning rates between the High Noise and Low

704 Noise conditions, learning rates in the two conditions also showed substantial shared variance

705 (Figure 5B).

\section{Reward processing}

707 We hypothesized that the availability or receipt of reward would incidentally increase

708 learning rate, in keeping with previous results from explicit prediction (Lee et al., 2020; McGuire

709 et al., 2014). That previous research showed that learning rate was elevated following rewarded

710 trials despite the fact that the reward did not provide additional information. However, we did

711 not find the same to be true in our experiment.

712 We also hypothesized that reward would alter oculomotor dynamics. Higher-value stimuli

713 tend to elicit saccades with higher peak velocity (Manohar et al., 2017; Takikawa, Kawagoe,

714 Itoh, Nakahara, \& Hikosaka, 2002) and shorter latency (Takikawa et al., 2002). However, none

715 of our selected oculomotor features (saccade latency, peak velocity, fixation duration, and

716 fixation gaze variability) showed effects of reward availability. It could be that the fast-paced

717 timing of our task (approximately $2 \mathrm{~s}$ per trial) caused participants to ignore reward information

718 in favor of consistent performance across trials. Future work will need to determine whether 
719 these differences represent meaningful distinctions between instructed and uninstructed contexts

720 or instead reflect other task features such as timing.

721 Irrespective of reward, we observed non-hypothesized patterns of decreasing saccade latency

722 and increasing peak velocity in higher-noise conditions. Greater peak velocity could relate to

723 larger prediction errors (and therefore larger saccades) in higher-noise blocks, but this does not

724 explain the finding of shortening latencies. The finding suggests that although participants could

725 not plan the saccade trajectory prior to stimulus appearance, they exhibited some ability to

726 prepare for the saccade execution.

727 Conclusions

728 We found that oculomotor behavior carried information about both the central tendency and

729 precision of internal predictive distributions during an uninstructed, gaze-based spatial prediction

730 task. Predictions were updated using a learning rate that was adapted to environmental statistics

731 across task blocks, but did not show the expected fine-scale trial-level adaptation previously

732 reported for overt predictions. Gaze variability during the pre-stimulus interval was associated

733 with the theoretical level of predictive precision.

734 Our results support a view that dynamic predictive inference is a general aspect of cognition,

735 not merely an esoteric pattern that emerges in response to specific experimental instructions. Our

736 findings on gaze variability suggest predictive representations carry information about the width

737 of predictive probability distributions even at the single-event scale, and that distributional

738 information is not driven solely by inter-event or inter-individual variance. The manifestation of

739 predictive uncertainty in gaze variability might reflect either a high-level strategy of sampling

740 the subjective belief distribution or a low-level side-effect of broadening the covert attentional

741 field. The lack of evidence for trial-level learning rate modulation suggests a scenario in which 
742 belief uncertainty interacts with structural inferences about the environment to modulate

743 learning. Investigating how people infer an environment's generative structure from sequences of

744 experiences, and how structural inferences guide the interpretation of subsequent feedback, is a

745 critical avenue for future work.

746 Context of the Research

747 This research began with the observation that the visual system has been a useful testbed for 748 many theories of cognition, including decision making. We broadly hypothesized that since gaze

749 is often predictive, it might be possible to emulate prior research on explicit predictive inference

750 in a more naturalistic manner using spatial prediction and eye tracking. The results suggest that

751 using spatial prediction and the visual system to answer fundamental questions about internal

752 representations of uncertainty is a fruitful avenue for future behavioral, computational, and

753 human neuroscience studies. Exploring biases and flexibility in spontaneous belief updating will

754 be important future directions, as will investigations of the underlying mechanisms.

756 Author contributions: LB and JTM contributed to experimental design. LB collected the data and

757 performed the analysis. LB prepared the manuscript with assistance from JTM.

\section{GRANTS}

760 This work was supported by National Science Foundation grants 1755757 and 1809071,

761 National Eye Institute F32 award EY029134, Office of Naval Research MURI award N00014-

762 16-1-2832 and DURIP award N00014-17-1-2304, and the Center for Systems Neuroscience

763 Postdoctoral Fellowship at Boston University. 


\section{ACKNOWLEDGEMENTS}

766 We thank Matthew Nassar, Sam Ling, Michele Rucci, and David Somers for helpful discussion.

767 We gratefully acknowledge support for acquisition of eye-tracking equipment from Barbara

768 Shinn-Cunningham and Boston University’s Center for Research in Sensory Communication and

769 Emerging Neural Technology. 


\section{REFERENCES}

771 Bakst, L., \& McGuire, J. (2019, July 18). Gaze variability as a measure of subjective uncertainty. Retrieved from osf.io/admsb

773

774

775

776

777

778

779

780

781

782

783

784

785

786

787

788

789

790

791

792

Behrens, T. E. J., Woolrich, M. W., Walton, M. E., \& Rushworth, M. F. S. (2007). Learning the value of information in an uncertain world. Nature Neuroscience, 10(9), 1214-1221. https://doi.org/10.1038/nn1954

Bonawitz, E., Denison, S., Griffiths, T. L., \& Gopnik, A. (2014). Probabilistic models, learning algorithms, and response variability: sampling in cognitive development. Trends in Cognitive Sciences, 18(10), 497-500. https://doi.org/10.1016/j.tics.2014.06.006

Cavanagh, J. F., Wiecki, T. V, Kochar, A., \& Frank, M. J. (2014). Eye tracking and pupillometry are indicators of dissociable latent decision processes. Journal of Experimental Psychology. General, 143(4), 1476-1488. https://doi.org/10.1037/a0035813

Clark, A. (2013). Whatever next? Predictive brains, situated agents, and the future of cognitive science. Behavioral and Brain Sciences, 36(3), 181-204. https://doi.org/10.1017/S0140525X12000477

Corrado, G. S., Sugrue, L., Seung, H. S., \& Newsome, W. T. (2005). Linear-nonlinear-poisson models of primate choice dynamics, 84(3), 581-617. https://doi.org/10.1901/jeab.2005.2305

D’Acremont, M., \& Bossaerts, P. (2016). Neural Mechanisms behind Identification of Leptokurtic Noise and Adaptive Behavioral Response. Cerebral Cortex, 26(4), 1818-1830. https://doi.org/10.1093/cercor/bhw013

Diaz, G., Cooper, J., \& Hayhoe, M. (2013). Memory and prediction in natural gaze control. Phil Trans $R$ Soc B, 368(20130064), 1-9. Retrieved from 
http://dx.doi.org/10.1098/rstb.2013.0064

Griffiths, T. L., \& Tenenbaum, J. B. (2006). Optimal Predictions in Everyday Cognition. Psychological Science, 17(9), 767-773.

Hafed, Z. M., Lovejoy, L. P., \& Krauzlis, R. J. (2011). Modulation of Microsaccades in Monkey

Hayhoe, M., \& Ballard, D. (2005). Eye movements in natural behavior. Trends in Cognitive Sciences, 9(4), 188-194. https://doi.org/10.1016/j.tics.2005.02.009

801

802

803

804

805

806

807

808

809

810

811

812

813

814 during a Covert Visual Attention Task. Journal of Neuroscience, 31(43), 15219-15230. https://doi.org/10.1523/JNEUROSCI.3106-11.2011

Hayhoe, M. M., Mckinney, T., Chajka, K., \& Pelz, J. B. (2012). Predictive eye movements in natural vision. Experimental Brain Research, 217, 125-136. https://doi.org/10.1007/s00221-011-2979-2

Henderson, J. M. (2017). Gaze Control as Prediction. Trends in Cognitive Sciences, 21(1), 1523. https://doi.org/10.1016/j.tics.2016.11.003

Knill, D. C., \& Pouget, A. (2004). The Bayesian brain : the role of uncertainty in neural coding and computation. Trends in Neurosciences, 27(12), 712-719. https://doi.org/10.1016/j.tins.2004.10.007

Konovalov, A., \& Krajbich, I. (2016). Gaze data reveal distinct choice processes underlying model-based and model-free reinforcement learning. Nature Communications, 7(12438), 111. https://doi.org/10.1038/ncomms 12438

Kording, K. P., \& Wolpert, D. M. (2004). Bayesian integration in sensorimotor learning. Nature, 427, 244-247.

Krajbich, I., Armel, C., \& Rangel, A. (2010). Visual fixations and the computation and comparison of value in simple choice. Nature Neuroscience, 13(10), 1292-1298. 
https://doi.org/10.1038/nn.2635

817 Land, M. F., \& McLeod, P. (2000). From eye movement to actions: how batsman hit the ball. $818 \quad$ Nature Neuroscience, 3(12), 1340-1345.

819 Lee, S., Gold, J. I., \& Kable, J. W. (2020). The human as delta-rule learner. Decision, 7(1), 55820 66. https://doi.org/10.1037/dec0000112

821 Luo, T. Z., \& Maunsell, J. H. R. (2018). Attentional Changes in Either Criterion or Sensitivity 822 823 Are Associated with Robust Modulations in Lateral Prefrontal Cortex. Neuron, 97(6),

McGuire, J. T., Nassar, M. R., Gold, J. I., \& Kable, J. W. (2014). Functionally Dissociable 828 829 1382-1393. https://doi.org/10.1016/j.neuron.2018.02.007

Manohar, S. G., Finzi, R. D., Drew, D., \& Husain, M. (2017). Distinct Motivational Effects of Contingent and Noncontingent Rewards. Psychological Science, 28(7), 1016-1026. https://doi.org/10.1177/0956797617693326

Nandy, A. S., Nassi, J. J., \& Reynolds, J. H. (2017). Laminar Organization of Attentional Modulation in Macaque Visual Area V4. Neuron, 93(1), 235-246. https://doi.org/10.1016/j.neuron.2016.11.029

Nassar, M. R., Bruckner, R., Gold, J. I., Li, S.-C., Heekeren, H. R., \& Eppinger, B. (2016). Age differences in learning emerge from an insufficient representation of uncertainty in older

836 Nassar, M. R., Bruckner, R., \& Frank, M. J. (2019). Statistical context dictates the relationship 837 between feedback-related EEG signals and learning. ELife, 8, 1-26.

838 https://doi.org/10.7554/eLife.46975 
Nassar, M. R., McGuire, J. T., Ritz, H., \& Kable, J. W. (2019). Dissociable Forms of Uncertainty-Driven Representational Change Across the Human Brain. Journal of Neuroscience, 39(9), 1688-1698.

Nassar, M. R., Rumsey, K. M., Wilson, R. C., Parikh, K., Heasly, B., \& Gold, J. I. (2012). Rational regulation of learning dynamics by pupil-linked arousal systems. Nature Neuroscience, 15(7), 1040-1046. https://doi.org/10.1038/nn.3130

Nassar, M. R., Wilson, R. C., Heasly, B., \& Gold, J. I. (2010). An approximately Bayesian deltarule model explains the dynamics of belief updating in a changing environment. The Journal of Neuroscience: The Official Journal of the Society for Neuroscience, 30(37), 12366-12378. https://doi.org/10.1523/JNEUROSCI.0822-10.2010

O’Reilly, J. X., Schüffelgen, U., Cuell, S. F., Behrens, T. E. J., Mars, R. B., \& Rushworth, M. F. S. (2013). Dissociable effects of surprise and model update in parietal and anterior cingulate cortex. Proceedings of the National Academy of Sciences of the United States of America, 110(38), E3660-9. https://doi.org/10.1073/pnas.1305373110

Ossmy, O., Moran, R., Pfeffer, T., Tsetsos, K., Usher, M., \& Donner, T. H. (2013). The Timescale of Perceptual Evidence Integration Can Be Adapted to the Environment. Current Biology, 23(11), 981-986. https://doi.org/10.1016/j.cub.2013.04.039

Payzan-Lenestour, E., \& Bossaerts, P. (2011). Risk, unexpected uncertainty, and estimation uncertainty: Bayesian learning in unstable settings. PLoS Computational Biology, 7(1). https://doi.org/10.1371/journal.pcbi.1001048

Payzan-LeNestour, E., Dunne, S., Bossaerts, P., \& O’Doherty, J. (2013). The Neural Representation of Unexpected Uncertainty during Value-Based Decision Making. Neuron, 79(1), 191-201. https://doi.org/10.1016/j.neuron.2013.04.037 
862 Peirce, J. (2007). PsychoPy - Psychophysics software in Python. J Neurosci Methods, 162(1-2), $8-13$.

864 Poletti, M., \& Rucci, M. (2016). A compact field guide to the study of microsaccades:

865 Challenges and functions. Vision Research, 118, 83-97.

$866 \quad$ https://doi.org/10.1016/j.visres.2015.01.018

867 Shimojo, S., Simion, C., Shimojo, E., \& Scheier, C. (2003). Gaze bias both reflects and

868 influences preference. Nature Neuroscience, 6(12), 1317-1322.

869 https://doi.org/10.1038/nn1150

870 Stocker, A. A., \& Simoncelli, E. P. (2006). Noise characteristics and prior expectations in human

$871 \quad$ visual speed perception. Nature Neuroscience, 9(4), 578-585.

872 https://doi.org/10.1038/nn1669

873 Summerfield, C., \& De Lange, F. P. (2014). Expectation in perceptual decision making: Neural 874 and computational mechanisms. Nature Reviews Neuroscience, 15(11), 745-756. https://doi.org/10.1038/nrn3838

Takikawa, Y., Kawagoe, R., Itoh, H., Nakahara, H., \& Hikosaka, O. (2002). Modulation of saccadic eye movements by predicted reward outcome. Experimental Brain Research, 142,

882 von Trapp, G., Buran, B. N., Sen, K., Semple, M. N., \& Sanes, D. H. (2016). A Decline in 883 Response Variability Improves Neural Signal Detection during Auditory Task Performance. 884 Journal of Neuroscience, 36(43), 11097-11106. https://doi.org/10.1523/JNEUROSCI.1302- 
886 Vul, E., Goodman, N., Griffiths, T. L., \& Tenenbaum, J. B. (2014). One and done? Optimal

887 decisions from very few samples. Cognitive Science, 38(4), 599-637.

$888 \quad$ https://doi.org/10.1111/cogs.12101

889 Vul, E., \& Pashler, H. (2008). Measuring the Crowd Within: Probabilistic Representations

$890 \quad$ Within Individuals. Psychological Science, 19(7), 645-647.

891 Yu, A. J., \& Dayan, P. (2005). Uncertainty, neuromodulation, and attention. Neuron, 46(4), 681892 692. https://doi.org/10.1016/j.neuron.2005.04.026

893 Yu, G., Xu, B., Zhao, Y., Zhang, B., Yang, M., Kan, J. Y. Y., ... Dorris, M. C. (2016).

894 Microsaccade direction reflects the economic value of potential saccade goals and predicts

895 saccade choice. Journal of Neurophysiology, 115(2), 741-751.

896 https://doi.org/10.1152/jn.00987.2015

897 Yuval-Greenberg, S., Merriam, E. P., \& Heeger, D. J. (2014). Spontaneous microsaccades reflect shifts in covert attention. The Journal of Neuroscience, 34(41), 13693-13700.

899 https://doi.org/10.1523/JNEUROSCI.0582-14.2014

900 